\title{
Search for Galaxies Clusters around Radio Galaxies with Different Linear Sizes
}

\author{
G. A. Ohanian \\ NAS RA V. Ambartsumian Byurakan Astrophysical Observatory (BAO), Armenia \\ E-mail: gohanian@bao.sci.am
}

\begin{abstract}
In order to understand how galaxies have formed and evolved in dense environments, and how these environments themselves evolve, we use the method of optical photometric survey of the field around radio galaxies. For this purpose, a statistical significant sample of radio galaxies consisting of 3 size radio source subclasses - GPS, CSS and FRI/II is selected for observations at the $1 \mathrm{~m}$ Schmidt telescope of the Byurakan Astrophysical Observatory. This method will allow us not only to detect distant clusters of galaxies, but also watch the sequence of development of these clusters - how changes their compactness, richness, and so on.
\end{abstract}

Keywords: Radio Galaxies - Galaxies Clusters - Galaxies Formation - Galaxies Evolution - AGN.

\section{Introduction}

One of the main problems of extragalactic astronomy is the understanding how galaxies have formed and evolved. There are two basic approaches to solve this problem. One of which, that is widely accepted in the literature is so called standard scenario (e. g. Benson, 2010), and the other, which is less disseminated in the scientific literature, is Ambartsumian's concept (Ambartsumian 1958).

It is a fact that yet more than 60 years ago Ambartsumian (Ambartsumian 1958) stressed the role of AGN in galaxies formation and evolution. In recent years many works appear where importance of the role of AGN in galaxy formation and evolution have been revealed (e. g. Morganti et al. 2009). But the role of AGN in the above mentioned approaches is essentially different.

In the standard scenarios, it is believed that injection of matter and others types of activity are secondary phenomena, and a primarily phenomenon 
is the gravitationally binding energy released from accretion of diffuse matter onto galaxies nuclei, which contain super massive black holes (SMBHs), and is discussed the efficiency with which AGN can transfer energy to the surrounding gas.

According to Ambartsumian's concept, the source of energy is effect of interior property of nuclei of galaxies, and a mechanism by which it can be transferred is still open.

Essentially, a key question, which arises when clear up a problem of formation and evolution of galaxies, irrespective of approaches, is the question of energy: what is the source of energy of AGN and its budget owing to formed galaxies and provide its subsequent development?

The main aim of our project is clear up this question. Our research is based not on preconceived opinion, but mainly on the observational data. When analyzing observational data, one bears in mind the following circumstance: the state of a galaxy is to be explained in terms of the entire activity of the nucleus over the preceding period that is the whole history of the nucleus. This means that the state of the galaxy should correlate with the present state of the nucleus (Ambartsumian 1965). These data, particularly collected during the last 10-15 years are the following:

- determination of the correlation of the integral parameters of the nuclei with those of the galaxies (e. g. Ferrarese \& Merritt 2000),

- collected data about stellar populations of galaxies galaxies (e. g. Hicks et al. 2010),

- growing evidence for the AGN intermittent activity (e. g. Saikia \& Jamrozy 2010,

- evolution of radio sources (e. g. O'Dea and Baum 1997).

Research should be made, including a thorough analysis of the abovementioned factual material and by correctly summarizing the results obtained. It will allow us to discover the patterns of galaxies development and approach the problem of their origin (even with one step). In addition to the mentioned research, bellow is presented an observational program that can be implementing with medium and large telescopes. 


\section{Search for Galaxies Clusters Around Radio Galax- ies with Different Linear Sizes. Objectives and sample selection}

Clusters represent the largest gravitationally bound and densest structures in the universe. As such, they provide a unique context for studying the formation of both galaxies and large scale structure. In order to understand how galaxies form and evolve in dense environments, and how these environments themselves evolve, it is important to trace the origins of galaxy clusters.

Many studies show that radio quiet AGN, which are usually associated with less massive spiral galaxies, are less clustered than radio loud AGN, which are associated with more massive elliptical galaxies (e. g. Falder et al. 2010). So, the method of detecting cluster of galaxies around radio galaxies is very effective (e. g. Miley \& De Breuck 2008).

The sizes of the most powerful radio emitters in the Universe vary from less than $1 \mathrm{pc}$ to more than $1 \mathrm{Mpc}$. This large range of sizes has been interpreted as evidence for the evolution of the linear sizes of radio structure (e. g. O'Dea \& Baum 1997). A crucial element in the study of their evolution is the identification of the young compact counterparts of "old" FRI/FRII (B. L. Fanaroff \& J. M. Riley 1974) extended objects. Good candidates for young radio sources are those with peaked spectra (Gigahertz Peaked Spectrum - GPS, linear sizes $<1 \mathrm{kpc}$ and Compact Steep Spectrum - CSS, linear sizes between $1 \mathrm{kpc}$ and $20 \mathrm{kpc}$. In the general scenario of the evolution of powerful radio loud AGNs, GPS sources evolve into CSS sources and these into super galactic-size FR I or FR II objects. The dynamic evolution of the double-lobed radio sources, characterized by the total extent of the source, advance speed of the hotspots and the dependence of the density distribution of the interstellar and intergalactic medium along the way of the propagating jets and lobes, predicts the increase of the radio power with the linear size of the source in the GPS and CSS phase until they reach the 1-3 kpc size. Then the larger CSS objects should start to slowly decrease their luminosity but the sharp radio power decrease is visible only in the FR I and FR II phase of evolution (K. R. Kaiser \& P. N. Best, 2007). Finally, after the cut off of the material supply to the central engine of the galaxy, the sources begin their fading phase. They can come back on the main evolutionary sequence after the re-ignition of the radio activity (C. Konar et al. 2012).

If it is true that GPS and CSS sources are young version of the large radio sources, it means that large scale radio sources host galaxies must live GPS-CSS phase. Therefore, the method of detecting the cluster of galaxies around radio galaxies with different linear sizes will enable not only discover clusters of galaxies, but also watch the sequence of development of these clusters - how changes their compactness, richness, and so on. 
For this purpose, we selected from literature four type of radio galaxies:

- GPS radio sources, linear sizes are within the range of galactic nuclei,

- CSS radio sources, linear sizes are within the optical dimensions of the galaxies,

- FR II and FR I radio sources, linear sizes are larger in optical dimensions of the galaxy and can reach up to several Mpc.

\section{Research Methods}

An efficient technique for finding clusters of galaxies is an optical narrowband survey of the field around radio galaxies. This observational program can be realized with modernized $1 \mathrm{~m}$ Schmidt telescope of the Byurakan Astrophysical Observatory of the National Academy of Sciences of Armenia (Movsesian et al. 2018). The $4 \mathrm{k} \times 4 \mathrm{k}$ Apogee (USA) liquid-cooled CCD with RON 11.1e-, a pixel size of 0.868 arcsec, and field of view of about 1 degree was mounted in the focus of the telescope. The detector is equipped with a turret bearing 20 intermediate-band filters (FWHM = $250 \mathrm{~A}$ ) uniformly covering the 4000-9000 A wavelength range, five broadband filters (SDSS $\mathrm{u}$, $\mathrm{g}, \mathrm{r}, \mathrm{i}, \mathrm{z})$ and three narrow-band filters $(5000 \mathrm{~A}, 6560 \mathrm{~A}$ and $6760 \mathrm{~A}, \mathrm{FWHM}$ $=100 \mathrm{~A})$.

\section{Expected Results and Summary}

The method of detecting the cluster of galaxies around radio galaxies with different linear sizes will enable us not only discover clusters of galaxies, but also watch the sequence of development of these clusters. Averaging richness, compactness of clusters of galaxies over the size subclasses enables us to investigate correlations between mean properties of clusters of galaxies and radio sources sizes.

To specify in more concrete terms, we expect to obtain in the course of this project the following results - photometry of galaxies, obtaining their photometric redshifts, estimating richness and compactness of clusters of galaxies. So, this data will enable not only discover clusters of galaxies, but also watch the sequence of development of these clusters - how changes their compactness, richness, and so on.

This project is open for collaboration. 


\section{References}

Ambartsumian, V. A., 1958, La Structure et l'Evolution de 'Univers, Editions Stoops, Bruxelles, 241

Ambartsumian, V. A., 1965, The Structure and Evolution of Galaxies, Interscience Publishers, 1

Benson, A. J., arXiv: astro-ph/10065394

Best et al., 2007, MNRAS, 379, 894

Burns, J. O., 1990, AJ, 99, 14

Falder, J. T., Stevens, J. A. et al., 2010, MNRAS, 405, 347

Fanaroff, B. L., Riley, J. M., 1974, MNRAS, 167P, 31

Ferrarese, L., Merritt, D. 2000, ApJ, 539, 9

Hicks, A. K., Mushotzky, R., Donahue, M., 2010, ApJ, 719, 1844

Kaiser, K. R., Best, P. N., 2007, MNRAS, 381, 1548

Konar, C., et. al., 2012, MNRAS, 424, 1061

Miley G. K. and De Breuck, C., 2008, AARv,15, 67, 2008

Movsessian, T. A., Dodonov, S. N., Gabrielyan, V. V., Kotov, S. S., Gevorgyan, M., 2017, ComBAO, V. 1, 92-101

O'Dea, C. P., 1998, PASP, 110, 493, 1998

Overzier, R. A. et al., 2009, ApJ, 704, 548

Saikia J. A., Jamrozy M., arXiv:astro-ph/1004.0640. 